\title{
SELF-APPROXIMATION OF HURWITZ ZETA-FUNCTIONS
}

\author{
RAMŪNAS GARUNKŠTis, ERIKAS KarIKOVAS
}

\begin{abstract}
We are looking for real numbers $\alpha$ and $d$ for which there exist "many" real numbers $\tau$ such that the shifts of the Hurwitz-zeta function $\zeta(s+i \tau, \alpha)$ and $\zeta(s+i d \tau, \alpha)$ are 'near' each other.
\end{abstract}

Keywords: Hurwitz zeta-function, strong recurrence, universality theorem.

\section{Introduction}

Let $s=\sigma+i t$ denote a complex variable. For $\sigma>1$, the Hurwitz zeta-function is given by

$$
\zeta(s, \alpha)=\sum_{n=0}^{\infty} \frac{1}{(n+\alpha)^{s}},
$$

where $\alpha$ is a parameter from the interval $(0,1]$. The Hurwitz zeta-function can be continued analytically to the entire complex plane except for a simple pole at $s=1$. For $\alpha=1$ we get $\zeta(s, 1)=\zeta(s)$, where $\zeta(s)$ is the Riemann zeta-function.

In this paper we consider the following problem. Find all real numbers $0<$ $\alpha \leqslant 1$ and $d$ such that, for any compact subset $\mathcal{K}$ of the strip $1 / 2<\sigma<1$ and any $\varepsilon>0$,

$$
\liminf _{T \rightarrow \infty} \frac{1}{T} \operatorname{meas}\left\{\tau \in[0, T]: \max _{s \in \mathcal{K}}|\zeta(s+i \tau, \alpha)-\zeta(s+i d \tau, \alpha)|<\varepsilon\right\}>0,
$$

where meas $A$ stands for the Lebesgue measure of a measurable set $A$. This problem is motivated by Bagchi $[1,2,3]$ result that the Riemann hypothesis for the Riemann zeta-function is valid if and only if the inequality (1) is valid for $\alpha=1$ and $d=0$. In the case of the Riemann zeta-function $(\alpha=1)$ the inequality (1) was proved by Nakamura [11] for all algebraic irrational $d$, afterwards by Pańkowski [14] nia.

The first author is supported by grant no MIP-066/2012 from the Research Council of Lithua-

2010 Mathematics Subject Classification: primary: 11M35; secondary: 11M26 
for all irrational $d$, and recently by Nakamura and Pańkowski [13] for $0 \neq d=a / b$ with $|a-b| \neq 1, \operatorname{gcd}(a, b)=1$ (the papers [5, 12], where non-zero rational $d$ were considered, contain a gap in the proof of the main theorem, see [13]). The case, when $\alpha \neq 1 / 2,1$ is a rational or transcendental number and $d=0$, is a partial case of the universality theorem for the Hurwitz zeta-function which is proved independently by Bagchi [1] and Gonek [7], see also [9]. More on the universality theorems see books of Laurinčikas [8], Steuding [15], and the survey of Matsumoto [10]. Here we will prove the case then $\alpha$ is a transcendental number and $d$ is a rational number, we will also show that for any transcendental number $\alpha$ the inequality (1) is true for almost all numbers $d$ and that for any irrational number $d$ the inequality (1) is true for almost all numbers $\alpha$. Next we state our results more precisely.

Let $d_{1}, d_{2}, \ldots, d_{k}, \alpha$ be real numbers and let $\alpha$ be a transcendental number from the interval $(0,1]$.

Let

$$
A\left(d_{1}, d_{2}, \ldots, d_{k} ; \alpha\right)=\left\{d_{j} \log \left(n_{j}+\alpha\right): j=1, \ldots, k ; n_{j} \in \mathbb{N}_{0}\right\}
$$

be a multiset, where $\mathbb{N}_{0}$ denotes the set of all non-negative integers. Note that in a multiset the elements can appear more than once. For example $\{1,2\}$ and $\{1,1,2\}$ are different multisets, but $\{1,2\}$ and $\{2,1\}$ are equal multisets. If a multiset $A\left(d_{1}, d_{2}, \ldots, d_{k} ; \alpha\right)$ is linearly independent over rational numbers then $A\left(d_{1}, d_{2}, \ldots, d_{k} ; \alpha\right)$ is a set and the numbers $d_{1}, \ldots, d_{k}$ are linearly independent over $\mathbb{Q}$. We prove the following theorem.

Theorem 1. Let $l \leqslant m$ be positive integers and let $\alpha$ be a transcendental number from the interval $(0,1]$. Let $d_{1}, \ldots, d_{l} \in \mathbb{R}$ be such that $A\left(d_{1}, d_{2}, \ldots, d_{l} ; \alpha\right)$ is linearly independent over $\mathbb{Q}$. For $m>l$, let $d_{l+1}, \ldots, d_{m} \in \mathbb{R}$ be such that each $d_{k}, k=l+1, \ldots, m$ is a linear combination of $d_{1}, \ldots, d_{l}$ over $\mathbb{Q}$. Then

$$
\begin{aligned}
\liminf _{T \rightarrow \infty} \frac{1}{T} \text { meas }\{\tau & \in[0, T]: \\
& \left.\max _{1 \leqslant j, k \leqslant m} \max _{s \in \mathcal{K}}\left|\zeta\left(s+i d_{j} \tau, \alpha\right)-\zeta\left(s+i d_{k} \tau, \alpha\right)\right|<\varepsilon\right\}>0 .
\end{aligned}
$$

In the inequality (2), for almost all $\varepsilon$, 'lim inf' can be replaced by 'lim' similarly as in Theorem 2 of [5]. Note that for any transcendental number $\alpha, 0<\alpha \leqslant 1$, and for any real number $d_{1}$, the set $A\left(d_{1} ; \alpha\right)$ is linearly independent over $\mathbb{Q}$. The following propositions show that for any positive integer $l$ 'most' collections of real numbers $d_{1}, d_{2}, \ldots, d_{l}, \alpha$, where $0<\alpha \leqslant 1$, are such that $A\left(d_{1}, d_{2}, \ldots, d_{l} ; \alpha\right)$ is linearly independent over $\mathbb{Q}$.

Proposition 2. Let $\alpha$ be a transcendental number and $l \geqslant 2$. If the set $A\left(d_{1}, d_{2}, \ldots, d_{l-1} ; \alpha\right)$ is linearly independent over $\mathbb{Q}$, then the set

$$
D=\left\{d_{l} \in \mathbb{R}: A\left(d_{1}, d_{2}, \ldots, d_{l} ; \alpha\right) \text { is linearly dependent over } \mathbb{Q}\right\}
$$

is countable. 
Proposition 3. Let $d_{1}, d_{2}, \ldots, d_{l}$ be real numbers linearly independent over $\mathbb{Q}$. Then the set

$$
B=\left\{\alpha \in(0,1]: A\left(d_{1}, d_{2}, \ldots, d_{l} ; \alpha\right) \text { is linearly dependent over } \mathbb{Q}\right\}
$$

is countable.

In the next section we prove Theorem 1 . Section 3 is devoted to proofs of Propositions 2 and 3.

\section{Proof of Theorem 1}

We follow the proof of Theorem 1 in [5]. Also lemmas from [5] will be used. As it was already mentioned the proof of Theorem 1 in [5] contains a gap, however here we avoid this gap because we work directly with $\zeta(s, \alpha)$ instead of $\log \zeta(s, \alpha)$.

Let us start with a truncated Hurwitz zeta-function

$$
\zeta_{v}(s, \alpha)=\sum_{q \leqslant v} \frac{1}{(q+\alpha)^{s}}
$$

By conditions of the theorem there are integers $a \neq 0$ and $a_{k, 1}, a_{k, 2}, \ldots, a_{k, l}$ such that

$$
d_{k}=\frac{1}{a}\left(a_{k, 1} d_{1}+a_{k, 2} d_{2}+\cdots+a_{k, l} d_{l}\right) \quad \text { for } \quad l<k \leqslant m
$$

Let

$$
A=\max _{l<k \leqslant m}\left\{\left|a_{k, 1}\right|+\left|a_{k, 2}\right|+\cdots+\left|a_{k, l}\right|\right\} .
$$

Denote by $\|x\|$ the minimal distance of $x \in \mathbb{R}$ to an integer. If

$$
\left\|\tau \frac{d_{n} \log (q+\alpha)}{2 \pi a}\right\|<\delta \quad \text { for } q \leqslant v \text { and } 1 \leqslant n \leqslant l
$$

then, by the relation (3),

$$
\left\|\tau \frac{d_{k} \log (q+\alpha)}{2 \pi}\right\|<A \delta \quad \text { for } q \leqslant v \text { and } l<k \leqslant m .
$$

By this and by the continuity in $s$ of the function $\zeta_{v}(s, \alpha)$ we have that for any $\varepsilon>0$ there is $\delta>0$ such that for $\tau$ satisfying (4)

$$
\max _{1 \leqslant k, n \leqslant m} \max _{s \in \mathcal{K}}\left|\zeta_{v}\left(s+i d_{k} \tau, \alpha\right)-\zeta_{v}\left(s+i d_{n} \tau, \alpha\right)\right|<\varepsilon .
$$

For positive numbers $\delta, v$, and $T$ we define the set

$$
S_{T}=S_{T}(\delta, v)=\left\{\tau: \tau \in[0, T],\left\|\tau \frac{d_{n} \log (q+\alpha)}{2 \pi a}\right\|<\delta, q \leqslant v, 1 \leqslant n \leqslant l\right\} .
$$


Let $U$ be an open bounded rectangle with vertices on the lines $\sigma=\sigma_{1}$ and $\sigma=\sigma_{2}$, where $1 / 2<\sigma_{1}<\sigma_{2}<1$, such that the set $\mathcal{K}$ is in $U$. Let $p>v$ be a positive integers. We have

$$
\begin{aligned}
\frac{1}{T} \int_{S_{T}} \int_{U} \sum_{k=1}^{m}\left|\zeta_{p}\left(s+i d_{k} \tau, \alpha\right)-\zeta_{v}\left(s+i d_{k} \tau, \alpha\right)\right|^{2} d \sigma d t d \tau \\
=\sum_{k=1}^{m} \int_{U} \frac{1}{T} \int_{S_{T}}\left|\zeta_{p}\left(s+i d_{k} \tau, \alpha\right)-\zeta_{v}\left(s+i d_{k} \tau, \alpha\right)\right|^{2} d \tau d \sigma d t .
\end{aligned}
$$

To evaluate the inner integrals of the right-hand side of the last equality we will apply Lemma 6 from [5]. By generalized Kronecker's theorem (see Lemma 5 in [5]) and by linear independence of $A\left(d_{1}, d_{2}, \ldots, d_{l} ; \alpha\right)$ the curve

$$
\omega(\tau)=\left(\tau \frac{d_{k} \log (q+\alpha)}{2 \pi a}\right)_{0 \leqslant q \leqslant p}^{1 \leqslant k \leqslant l}
$$

is uniformly distributed $\bmod 1$ in $\mathbb{R}^{l(p+1)}$. Let $R^{\prime}$ be a subregion of the $l(p+1)$ dimensional unit cube defined by inequalities

$$
\left\|y_{k, q}\right\| \leqslant \delta \quad \text { for } 1 \leqslant k \leqslant l \text { and } 0 \leqslant q \leqslant v
$$

and

$$
\left|y_{k, q}-\frac{1}{2}\right| \leqslant \frac{1}{2} \quad \text { for } 1 \leqslant k \leqslant l \text { and } v+1 \leqslant q \leqslant p .
$$

Let $R$ be a subregion of the $l(v+1)$-dimensional unit cube defined by inequalities

$$
\left\|y_{k, q}\right\| \leqslant \delta \quad \text { for } 1 \leqslant k \leqslant l \text { and } 0 \leqslant q \leqslant v
$$

Clearly meas $R^{\prime}=$ meas $R=(2 \delta)^{l(v+1)}$. Let

$$
\zeta_{p, v}\left(s+i d_{k} \tau, \alpha\right)=\zeta_{p}\left(s+i d_{k} \tau, \alpha\right)-\zeta_{v}\left(s+i d_{k} \tau, \alpha\right)
$$

Then in view of the linear dependence (3) we get

$$
\begin{aligned}
& \lim _{T \rightarrow \infty} \frac{1}{T} \int_{S_{T}} \sum_{k=1}^{m}\left|\zeta_{p, v}\left(s+i d_{k} \tau, \alpha\right)\right|^{2} d \tau \\
&=\lim _{T \rightarrow \infty} \frac{1}{T} \int_{S_{T}}\left(\sum_{k=1}^{l}\left|\zeta_{p, v}\left(s+i d_{k} \tau, \alpha\right)\right|^{2}\right. \\
&\left.+\sum_{k=l+1}^{m}\left|\zeta_{p, v}\left(s+\frac{i}{a}\left(a_{k, 1} d_{1}+a_{k, 2} d_{2}+\cdots+a_{k, l} d_{l}\right) \tau, \alpha\right)\right|^{2}\right) d \tau .
\end{aligned}
$$


By Lemma 6 in [5] and equality (7) we obtain that the last limit is equal to

$$
\begin{aligned}
& \int_{R^{\prime}}\left(\sum_{k=1}^{l}\left|\sum_{v<q \leqslant p} \frac{e^{-2 \pi i a y_{k, q}}}{(q+\alpha)^{s}}\right|^{2}\right. \\
& \left.+\sum_{k=l+1}^{m}\left|\sum_{v<q \leqslant p} \frac{e^{-2 \pi i\left(a_{k, 1} y_{1, q}+a_{k, 2} y_{2, q}+\cdots+a_{k, l} y_{l, q}\right)}}{(q+\alpha)^{s}}\right|^{2}\right) d y_{1,1} \ldots d y_{l, p} \\
& =\left.\operatorname{meas} R \int_{0}^{1} \ldots \int_{0}^{1}\left|\sum_{k=1}^{l}\right| \sum_{v<q \leqslant p} \frac{e^{-2 \pi i y_{k, q}}}{(q+\alpha)^{s}}\right|^{2} \\
& +\sum_{k=l+1}^{m} \mid \sum_{v<q \leqslant p} \frac{\left.\left.e^{-2 \pi i\left(a_{k, 1} y_{1, q}+a_{k, 2} y_{2, q}+\cdots+a_{k, l} y_{l, q}\right)}\right|^{2}\right)}{(q+\alpha)^{s}} d y_{1, v+1} \ldots d y_{l, p} \\
& =m \text { meas } R \sum_{v<q \leqslant p} \frac{1}{(q+\alpha)^{2 \sigma}} \ll \operatorname{meas} R \sum_{q>v} \frac{1}{(q+\alpha)^{2 \sigma}} .
\end{aligned}
$$

Consequently

$$
\begin{aligned}
\lim _{T \rightarrow \infty} \frac{1}{T} \int_{S_{T}} \int_{U} \sum_{k=1}^{m}\left|\zeta_{p}\left(s+i d_{k} \tau, \alpha\right)-\zeta_{v}\left(s+i d_{k} \tau, \alpha\right)\right|^{2} d \sigma d t d \tau & \\
& \ll \operatorname{meas} R \sum_{q>v} \frac{1}{(q+\alpha)^{2 \sigma_{1}}} .
\end{aligned}
$$

Again by Lemma 5 in [5],

$$
\lim _{T \rightarrow \infty} \frac{1}{T} \text { meas } S_{T}=\text { meas } R
$$

By (8) and (9), for large $v$, as $T \rightarrow \infty$, we have

$$
\begin{array}{r}
\text { meas }\left\{\tau: \tau \in S_{T}, \int_{U} \sum_{k=1}^{m}\left|\zeta_{p, v}\left(s+i d_{k} \tau, \alpha\right)\right|^{2} d \sigma d t<\sqrt{\sum_{q>v} \frac{1}{(q+\alpha)^{2 \sigma_{1}}}}\right\} \\
>\frac{1}{2} T \text { meas } R .
\end{array}
$$

Then Lemma 4 in [5] gives

$$
\begin{array}{r}
\operatorname{meas}\left\{\tau: \tau \in S_{T}, \max _{s \in \mathcal{K}} \sum_{k=1}^{m}\left|\zeta_{p, v}\left(s+i d_{k} \tau, \alpha\right)\right| \leqslant \frac{m}{d \sqrt{\pi}}\left(\sum_{q>v} \frac{1}{(q+\alpha)^{2 \sigma_{1}}}\right)^{\frac{1}{4}}\right\} \\
>\frac{1}{2} T \text { meas } R
\end{array}
$$


where $d=\min _{z \in \partial U} \min _{s \in \mathcal{K}}|s-z|$. Therefore we obtain that for any $\varepsilon>0$ there is $v=v(\varepsilon)$ such that for any $p>v$

$$
\begin{aligned}
\operatorname{meas}\left\{\tau: \tau \in S_{T}, \max _{s \in \mathcal{K}} \sum_{k=1}^{m}\left|\zeta_{p}\left(s+i d_{k} \tau, \alpha\right)-\zeta_{v}\left(s+i d_{k} \tau, \alpha\right)\right|\right. & <\varepsilon\} \\
& >\frac{1}{2} T \text { meas } R .
\end{aligned}
$$

Now we will prove that for any $\delta>0$ there is $p=p(\delta)$ such that

$$
\operatorname{meas}\left\{\tau: \max _{s \in \mathcal{K}} \sum_{k=1}^{m}\left|\zeta\left(s+i d_{k} \tau, \alpha\right)-\zeta_{p}\left(s+i d_{k} \tau, \alpha\right)\right|<\delta\right\}
$$

$$
>(1-\delta) T \text {. }
$$

The last formula together with (5), (6) and (10) yields Theorem 1 . We return to the proof of (11). By the mean value theorem of the Hurwitz zeta-function (see Garunkštis, Laurinčikas, and Steuding [6]) and by Carlson's Theorem (see Carlson [4]) we obtain

$$
\lim _{T \rightarrow \infty} \frac{1}{T} \int_{0}^{T}\left|\zeta(s+i x \tau, \alpha)-\zeta_{p}(s+i x \tau, \alpha)\right|^{2} d \tau=\sum_{q>p} \frac{1}{(q+\alpha)^{2 \sigma}},
$$

where $x$ is fixed. Thus (11) follows in view of

$$
\int_{0}^{T} \int_{U} \sum_{k=1}^{m}\left|\zeta\left(s+i d_{k} \tau, \alpha\right)-\zeta_{p}(s+i x \tau, \alpha)\right|^{2} d \sigma d t d \tau \ll T \sum_{q>p} \frac{1}{(q+\alpha)^{2 \sigma_{1}}} .
$$

Theorem 1 is proved.

\section{Proofs of Propositions 2 and 3}

Proof of Proposition 2. Let $\Omega$ be a set of all rational numbers sequences, where each sequence has only finitely many nonzero elements. Then $\Omega$ is a countable set. By $\mathbf{0}$ we denote the sequence all elements of which are zeros. Let $d_{1}=1$. Recall that the set $A(1 ; \alpha)$ is linearly independent. Then in view of the linear independence of $A\left(d_{1}, d_{2}, \ldots, d_{l-1} ; \alpha\right)$ we obtain that

$$
\begin{aligned}
& D=\left\{-\frac{d_{1} \sum_{n=0}^{\infty} a_{1 n} \log (n+\alpha)+\cdots+d_{l-1} \sum_{n=0}^{\infty} a_{l-1 n} \log (n+\alpha)}{\sum_{n=0}^{\infty} a_{l n} \log (n+\alpha)}:\right. \\
& \left(a_{10}, a_{11}, \ldots, a_{(l-1) 0}, a_{(l-1) 1}, \ldots, a_{l 0}, a_{l 1}, \ldots\right) \in \Omega \backslash \mathbf{0}, \\
& \left.\left(a_{l 0}, a_{l 1}, \ldots\right) \neq \mathbf{0}\right\} \text {. }
\end{aligned}
$$

Thus $D$ is a countable set. This proves the proposition. 
Proof of Proposition 3. We use the same notations as in the proof of Proposition 2. Similarly as before we have that

$$
\begin{aligned}
B=\left\{\alpha \in I: d_{1} \sum_{n=0}^{\infty} a_{1 n}\right. & \log (n+\alpha)+\cdots+d_{l} \sum_{n=0}^{\infty} a_{l n} \log (n+\alpha)=0, \\
& \left.\left(a_{10}, a_{11}, \ldots, a_{20}, a_{21}, \ldots, \ldots, a_{l 0}, a_{l 1}, \ldots\right) \in \Omega \backslash \mathbf{0}\right\} .
\end{aligned}
$$

Recall that $\Omega$ is a countable set. If, for fixed

$$
\left(a_{10}, a_{11}, \ldots, a_{20}, a_{21}, \ldots, \ldots, a_{l 0}, a_{l 1}, \ldots\right) \in \Omega \backslash \mathbf{0},
$$

the function

$$
f(\alpha)=d_{1} \sum_{n=0}^{\infty} a_{1 n} \log (n+\alpha)+\cdots+d_{l} \sum_{n=0}^{\infty} a_{l n} \log (n+\alpha)
$$

has only finite number of zeros in $(0,1]$, then the set $B$ is countable. Thus to prove the proposition it remains to show that $f(\alpha)$ has finitely many zeros in the interval $(0,1]$. In view of the condition that $d_{1}, d_{2}, \ldots, d_{k}$ are linearly independent and by the definition of $\Omega$ we have that there is a finite collection of real numbers $b_{0}, b_{1}, \ldots, b_{m}$, such that $b_{m} \neq 0$ and

$$
f(\alpha)=b_{0} \log (\alpha)+b_{1} \log (1+\alpha)+\cdots+b_{m} \log (m+\alpha) .
$$

Let $b_{n}, n \leqslant m$ be the first coefficient not equal to zero. Then we see that $f(\alpha)$ is unbounded in $(-n, 1 / 2)$ and is bounded in $(1 / 2,1]$. Thus $f(\alpha)$ is not a constant in $(-n, 1]$. Moreover there is a small positive number $\alpha_{0}$ such that $f(\alpha) \neq 0$ if $\alpha \in\left(-n,-n+\alpha_{0}\right)$. We consider $f(\alpha)$ as an analytic function in the half-plane $\Re \alpha>-n$ of the complex plane. A set of zeros of a non-constant analytic function is discrete. Thus there are finitely many zeros in the disc $|1-\alpha| \leqslant 1+n-\alpha_{0}$. We obtained that the function $f(\alpha)$ has finitely many zeros in $(0,1]$. This proves the proposition.

Acknowledgements. We thank the referee and Kohji Matsumoto for useful comments.

\section{References}

[1] B. Bagchi, The statistical behaviour and universality properties of the Riemann zeta-function and other allied Dirichlet series, PhD Thesis, Calcutta: Indian Statistical Institute, 1981.

[2] B. Bagchi, A joint universality theorem for Dirichlet L-functions, Math. Z. 181 (1982), 319-334.

[3] B. Bagchi, Recurrence in topological dynamics and the Riemann hypothesis, Acta Math. Hung. 50 (1987), 227-240. 
[4] F. Carlson, Contributions à la théorie des séries de Dirichlet. Note I., Arkiv för Mat., Astron. och Fysik 16 (1922), no. 18, 19 pp.

[5] R. Garunkštis, Self-approximation of Dirichlet L-functions, J. Number Theory 131 (2011), 1286-1295.

[6] R. Garunkštis, A. Laurinčikas, and J. Steuding, On the mean square of Lerch zeta-functions, Arch. Math. 80 (2003), 47-60.

[7] S.M. Gonek, Analytic Properties of Zeta and L-Functions, PhD Thesis, University of Michigan, 1979.

[8] A. Laurinčikas, Limit theorems for the Riemann zeta-function, Mathematics and its Applications (Dordrecht) 352. Dordrecht: Kluwer Academic Publishers, 1995.

[9] A. Laurinčikas, R. Garunkštis, The Lerch zeta-function, Kluwer, Dordrecht 2002

[10] K. Matsumoto, Probabilistic value-distribution theory of zeta-functions, Sugaku 53 (2001), 279-296 (in Japanese); English translation in Sugaku Expo. 17 (2004), 51-71.

[11] T. Nakamura, The joint universality and the generalized strong recurrence for Dirichlet L-functions, Acta Arith. 138 (2009), 357-362.

[12] T. Nakamura, The generalized strong recurrence for non-zero rational parameters, Archiv der Matematik 95 (2010), 549-555.

[13] T. Nakamura, Ł. Pańkowski, Self-approximation for Riemann zeta function, Bulletin of the Australian Mathematical Society 87 (2013), 452-461.

[14] Ł. Pańkowski, Some remarks on the generalized strong recurrence for L-functions, in: New Directions in Value Distribution Theory of zeta and L-Functions, Ber.Math., Shaker Verlag, Aachen, (2009), 305-315.

[15] J. Steuding, Value distribution of L-functions, Lecture Notes in Mathematics 1877, Springer, 2007.

Address: Ramūnas Garunkštis and Erikas Karikovas: Department of Mathematics and Informatics, Vilnius University, Naugarduko 24, 03225 Vilnius, Lithuania.

E-mail: ramunas.garunkstis@mif.vu.lt, erikas.karikovas@mif.stud.vu.lt

Received: 18 March 2013; revised: 30 September 2013 